Portland State University

PDXScholar

3-1-2019

\title{
Transformation of TFB2 Overexpression Plasmid into Hyperthermophile Pyrococcus furiosus COM1
}

\author{
Brent Marsonette \\ Portland State University
}

Follow this and additional works at: https://pdxscholar.library.pdx.edu/honorstheses

\section{Let us know how access to this document benefits you.}

\author{
Recommended Citation \\ Marsonette, Brent, "Transformation of TFB2 Overexpression Plasmid into Hyperthermophile Pyrococcus \\ furiosus COM1" (2019). University Honors Theses. Paper 663. \\ https://doi.org/10.15760/honors.676
}

This Thesis is brought to you for free and open access. It has been accepted for inclusion in University Honors Theses by an authorized administrator of PDXScholar. Please contact us if we can make this document more accessible: pdxscholar@pdx.edu. 
Transformation of TFB2 Overexpression Plasmid into Hyperthermophile Pyrococcus furiosus COM1

by

Brent Marsonette

An undergraduate honors thesis submitted in partial fulfillment of the requirements

of the degree of

Bachelor of Science

in

University Honors

and

Biology

Thesis Adviser

Michael S. Bartlett Ph.D

Portland State University 


\section{Abstract:}

Pyrococcus furiosus is an exceptional model organism for studies of life in extreme environments. Being a hyperthermophile, this archaeal species thrives at temperatures nearing that of boiling water. The underlying machinery for transcription in most archaeal species is poorly studied, and data concerning transcriptional regulation is limited. Transcription Factor B Subunit II (TFB2), a non-standard component of the transcription preinitiation complex in $P$. furiosus, is poorly understood regarding its function and mechanism. One hypothesis is that TFB2 is important for cellular response to oxidative stress. To test this, a plasmid that overexpresses the TFB2 protein was successfully transformed into $P$. furiosus COM1, as indicated by genetic isolation and PCR. Once successfully transformed, COM1 with excess TFB2 will be examined in order to see if its overexpression provides a protective effect against experimentally induced oxidative stress. These results will provide a baseline for future functional studies regarding the role of TFB2, ultimately furthering understanding of the underlying transcription machinery present within Pyrococcus furiosus and other archaea.

\section{Introduction:}

One of the best studied archaeal species is Pyrococcus furiosus, a hyperthermophilic archaeal species native to Vulcano, Italy. Archaea's distinction from other bacterial prokaryotic organisms was only discovered in the 1970s (Woese et al., 1977), and thus archaeal research may be considered to generally be in its early, exploratory stages for many areas of study. Hyperthermophiles are of biotechnological interest, showing promise for commercial use. Some 
important functionality derives from the proteins and mechanisms employed by hyperthermophiles that confer function and survival under what would be extremely stressful conditions for most other organisms. For a prominent example of this, in 1991, Lundberg et al. was able to isolate DNA Polymerase I from P. furiosus, demonstrating a novel 3'-5' proofreading ability during normal $P$. furiosus growth conditions. This DNA polymerase has since been integrated into modern PCR reactions in order to increase accuracy over the more traditional Taq-DNA polymerase (Kengen et al. 2017).

The experiments in this thesis aim to provide groundwork to further understanding regarding the cellular machinery that allows Pyrococcus furiosus to survive in otherwise unfavorable environments. More specifically, we will be investigating the ability of the anaerobic $P$. furiosus COM1 to adapt to oxidative stress. The non-standard general transcription factor TFB2 is likely to be important to $P$. furiosus, since it plays a role in vitro in formation of the preinitiation complex required for transcriptional activity in archaeal species and elsewhere in the kingdoms of life. However, its function in vivo in P. furiosus is currently unknown. Since deletion of the TFB2 gene causes enhanced sensitivity to oxidative stress, one hypothesis is that TFB2 may be critical in regulating genes responsible for survival under oxidizing conditions. This will be tested by transforming $P$. furiosus with DNA containing a gene for overexpression of the TFB2 subunit, and examining responses to oxidative stress. 


\section{Background:}

First described in 1986 by Fiala et al., P. furiosus has been shown to grow anaerobically at around $70-100^{\circ} \mathrm{C}$. P. furiosus has proven to be one of the most sought after model organisms for hyperthermophilic archaeal study, as the species' doubling rate in laboratory settings can be as rapid as 30 minutes. In addition, the organism is able to tolerate extremely high temperatures and anoxic environments while maintaining limited negative impacts on genetic or metabolic function (Fiala et al., 1986).

While wild-type $P$. furiosus has provided important basic information about the machinery of archaeal transcription, a novel mutant of $P$. furiosus, COM1, has proven to be better suited for laboratory experiments. The COM1 mutant has a naturally occurring tendency for DNA uptake, coupled with a pyrF gene deletion, providing a genetic selection strategy. COM1 was first documented by Lipscomb et al. in 2011 to remedy the lack of available mutants with selectable markers. The pyrF gene deletion allowed for accurate and simplified methods for the introduction of foreign DNA to living cells. Transforming DNA should contain the pyrF gene, allowing for growth in media that lacks uracil and/or relevant uracil precursors. $P$. furiosus COM1 also showed marked increases in DNA integration over previously used mutants of $P$. furiosus, with the authors noting the COM1 mutant was able to produce transformants in the range of $10^{5}$ per $\mu \mathrm{g}$ DNA introduced over a total of $10^{8}$ cells recovered per sample.

P. furiosus has displayed some intriguing responses in regards to overcoming less-thanoptimal environments. While the organism typically grows under anaerobic conditions, $P$. 
furiosus is able to modulate its metabolism in order to tolerate the presence of oxygen. Upwards of $8 \% \mathrm{O}_{2} \mathrm{vol} / \mathrm{vol}$ solutions have been experimentally identified to have no impact on cellular replication, and often only result in slightly decreased rates of metabolite formation $\left(\mathrm{H}_{2}\right.$, notably) (Thorgersen et al., 2012). Additionally, a paper by Williams et al. identified multiple pathways in which $P$. furiosus was able to tolerate varying levels of ionizing radiation with little effect on culture growth (Williams et al., 2007). While normally considered to be growing in one of the harshest environments within its natural habitat, P. furiosus demonstrates that it has extensive mechanisms present in its genetic code for diminishing the effects of a wide variety of potential stressors.

The plasmid that was chosen for this experiment was identified as "TFB2 id. 228", synthesized by Charles Wilson and Michael Bartlett, with a length of 7315 bp. TFB2 id. 228 has a pyrF positive selectable marker along with an additional copy of the gene encoding for TFB2, controlled by a constitutively active promoter. TFB2 has been identified to play an invaluable role in recruiting the preinitiation complex during cellular transcription in vitro. More specifically, it is hypothesized that the TFB2 protein plays a role in placement of the RNAP. However, a mechanistic understanding of TFB2 function is lacking, and the role of TFB2 in vivo is not yet understood (Micorescu et al., 2008). 


\section{Methods:}

In order to transform P. furiosus COM1, the cultures were initially grown within ideal temperature ranges of $80-90^{\circ} \mathrm{C}$. The more recently the cells have been grown, the better they displayed optimal transformation of varied plasmids, observable in preliminary experiments. Thus, new batches of COM1 were grown for each new transformation attempt. The growth media that $P$. furiosus was cultured in contained essential minerals, vitamins, and amino acids, as well as cellobiose as a carbon source. Uracil was added to media of wild-type COM1 in order to potentiate growth, because $P$. furiosus COM1 has no way of generating uracil on its own. All media were prepared using anaerobic techniques, and nitrogen gas was used in the headspace.

The media was prepared by first adding previously made 'base salts', 'minerals', and disodium/monosodium phosphate solutions at previously determined amounts, followed by the introduction of cellobiose mixed into a large flask. The solution was then heated to boiling, and was allowed to cool while $\mathrm{N}_{2}$ gas was bubbled generously through the solution. The cooled solution was then adjusted to ideal growing $\mathrm{pH}(6.8)$, and divided into separate tubes while sparging with $\mathrm{N}_{2}$ gas. The tubes were then sealed off using rubber stoppers in combination with aluminum caps and were autoclaved at $121^{\circ} \mathrm{C}$ for 20 minutes. The autoclaved tubes were then injected with amino acids, $125 \mathrm{mg} / \mathrm{mL}$ cystine, a vitamin solution (200x vitamins), and $2.5 \%$ $\mathrm{Na}_{2} \mathrm{~S}$ for reducing purposes. The growth tubes were then allowed to cool, followed by individual inoculation consisting of 500 $\mu 1$ previously grown COM1 cells. The COM1 cells were then incubated at $90^{\circ} \mathrm{C}$ for $24-48$ hours. 
Following growth, typically indicated by an increase in turbidity within the tube when compared to a control (Fig. 2), the cells were transformed using a TFB2 overexpression plasmid,

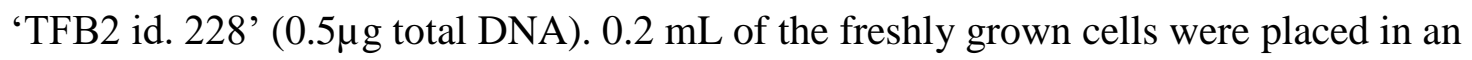
eppendorf tube with the addition of the aforementioned plasmid. The cells were then allowed to incubate for 5 minutes with occasional mixture from pipet tip insertion. The transformed COM1 cells were then transferred to previously created cellobiose roll tubes lacking uracil (Bartlett lab methods) and placed in the incubator at $85^{\circ} \mathrm{C}$ for $48-72$ hours. The tubes were inspected daily for colony growth, and were removed upon colony identification. The colonies were then removed under anoxic conditions, and placed into liquid growth media lacking uracil. The new uracilnegative growth tubes were then, again, placed into the incubator at $90^{\circ} \mathrm{C}$ for $24-48$ hours. The tubes were inspected daily, and removed once growth was identified.

Genetic isolation was originally attempted via methods developed for bacterial cultures, however, these methods proved to be unsuccessful. Once growth of the transformed $P$. furiosus was identified, the transformed DNA was isolated by a genome isolation protocol, employing phenol-chloroform extraction coupled with ethanol precipitation. To do this, the cell culture was first centrifuged in order to isolate the cellular mass from solution, and then suspended in a buffer solution containing Tris-HCl, EDTA, and sucrose. The suspension was then combined with a lysis solution comprised of guanidinium $\mathrm{HCl}$, and subsequently incubated for a few minutes. Following incubation, the sample was removed from the heat source and phenolchloroform was added. The sample was then mixed, and the aqueous layer was removed. The phenol-chloroform process was then repeated two more times on the same samples. The DNA was recovered from solution by standard ethanol precipitation. After precipitation, the resulting 
DNA pellet was washed with $70 \%$ ethanol and allowed to air dry for 5 minutes. Once dried, the samples were dissolved into $\mathrm{ddH}_{2} \mathrm{O}$ for storage at $-20^{\circ} \mathrm{C}$.

Samples were then tested for the presence of the plasmid by PCR. The primers used for identification of plasmid transformation were TGPForward and gdhR. After the PCR reaction was completed, resulting samples were examined for DNA bands by gel electrophoresis.Two lanes were designated for TFB2 at varied concentrations $(2 \mu \mathrm{L}$ and $20 \mu \mathrm{L})$, which were compared against an "O'GeneRuler $1 \mathrm{~kb}$ DNA Ladder". The gel was then analyzed and images were taken using an ultraviolet gel imaging device.

\section{$\underline{\text { Results: }}$}

To test the effect of TFB2 overexpression on $P$. furiosus cell physiology, it was necessary to transform cells with plasmid using a pyrF gene as a selectable marker. This has never previously been achieved in $P$. furiosus. The experiment was successful in transforming the TFB2 id. 228 overexpression plasmid into COM1 P. furiosus. The transformants were first identified in uracil negative media, as indicated in Figure 1. The uracil negative roll tubes provided to be effective methods for initial anaerobic transformation.

Successful growth of $P$. furiosus COM1 containing the plasmid was observed using uracil negative media on multiple occasions, indicating consistent transformation of the TFB2 id. 228 plasmid. The growing cells in liquid cultures could be observed as in Figure 2, with marked increases in turbidity (from cells in suspension) when looking through the tubes. 
As indicated by Figure 3, PCR products of the transformation were identified at exactly the expected length of the transformed plasmid, indicating that transformation had occurred (Fig. 3). The band indicating a $2 \mu \mathrm{L}$ concentration was perceived as a brighter indication of transformation when compared to the $20 \mu \mathrm{L}$ band. The product produced by the PCR protocol appeared on the gel between the $1000 \mathrm{bp}$ and $1500 \mathrm{bp}$ indicators produced by the $1 \mathrm{~kb}$ ladder, indicating that the DNA fragment was of the expected length - $1224 \mathrm{bp}$. This result indicated that plasmid DNA was successfully recovered from the transformed P. furiosus strain.

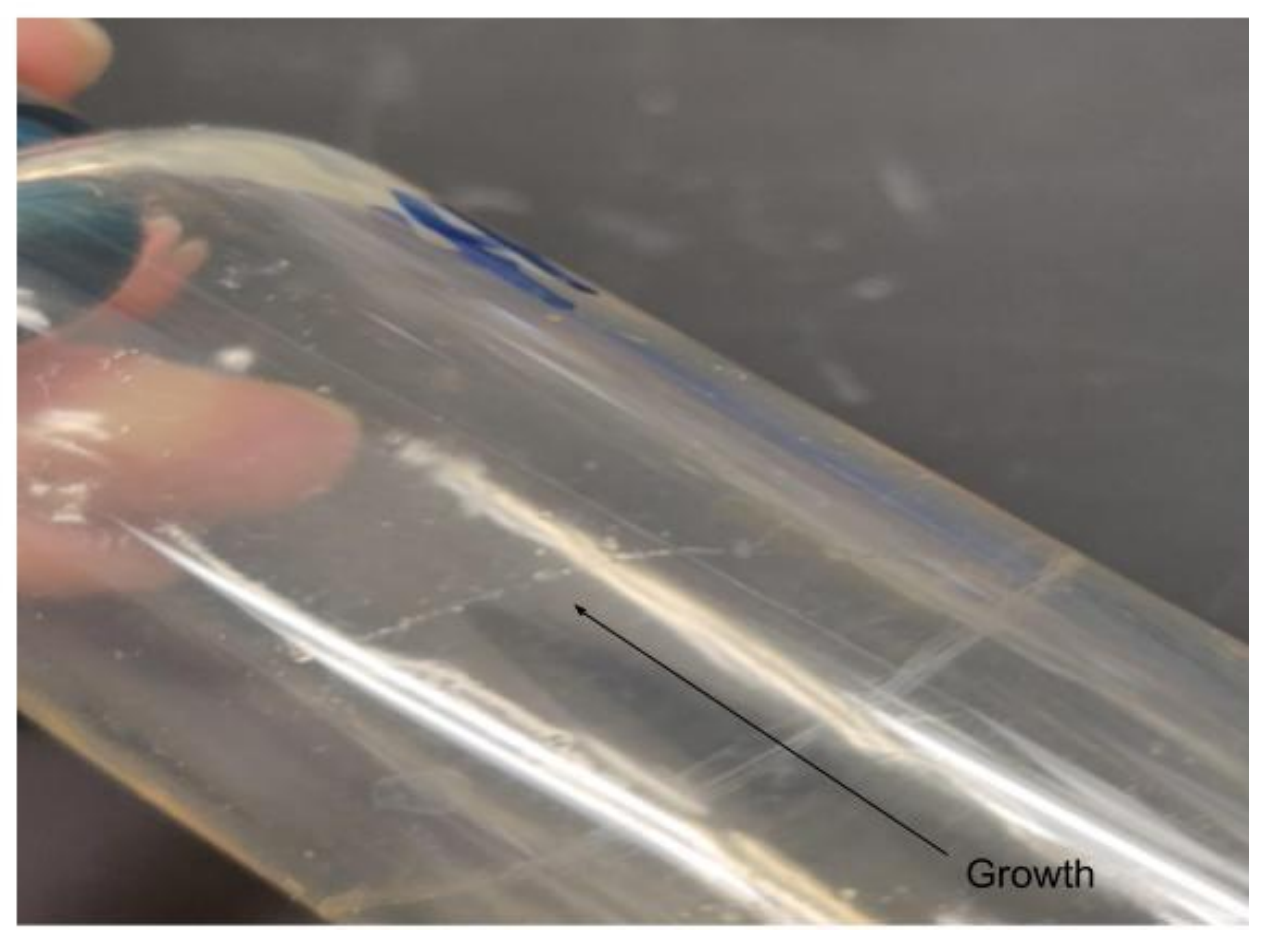

Figure 1: Transformed COM1 Growth. Present at the arrow above are colonies of growth present within an anaerobic roll tube lacking uracil. The colonies grew after 72 hours of incubation at $85^{\circ} \mathrm{C}$. 


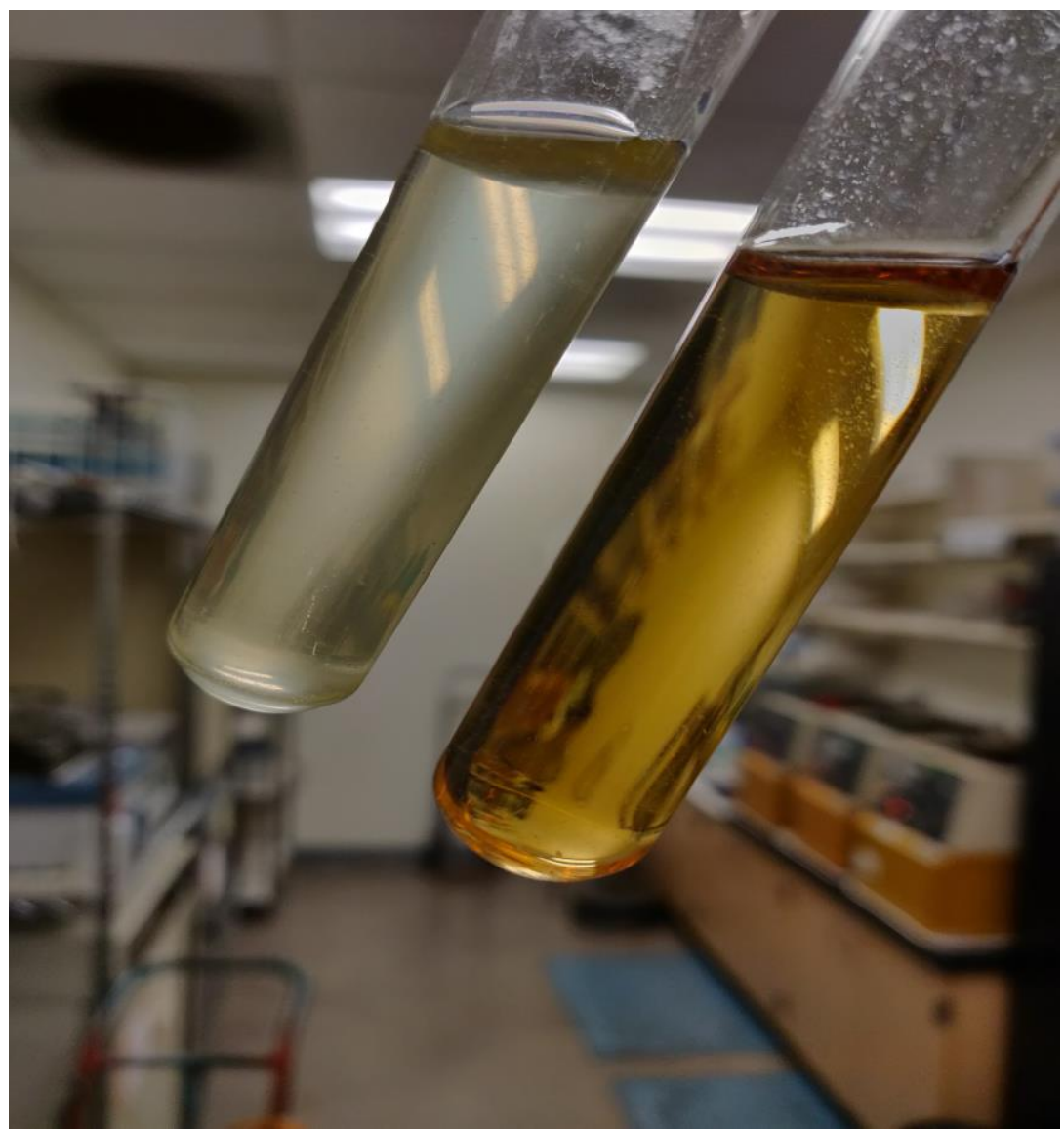

Figure 2: Growth Visualization of Pyrococcus furiosus COM1. Increased turbidity from cellular growth can be observed. Leftmost tube has been incubated for 24 hours with COM1 inoculation present. The tube on the right is a control which has been incubated for the same amount of time. 


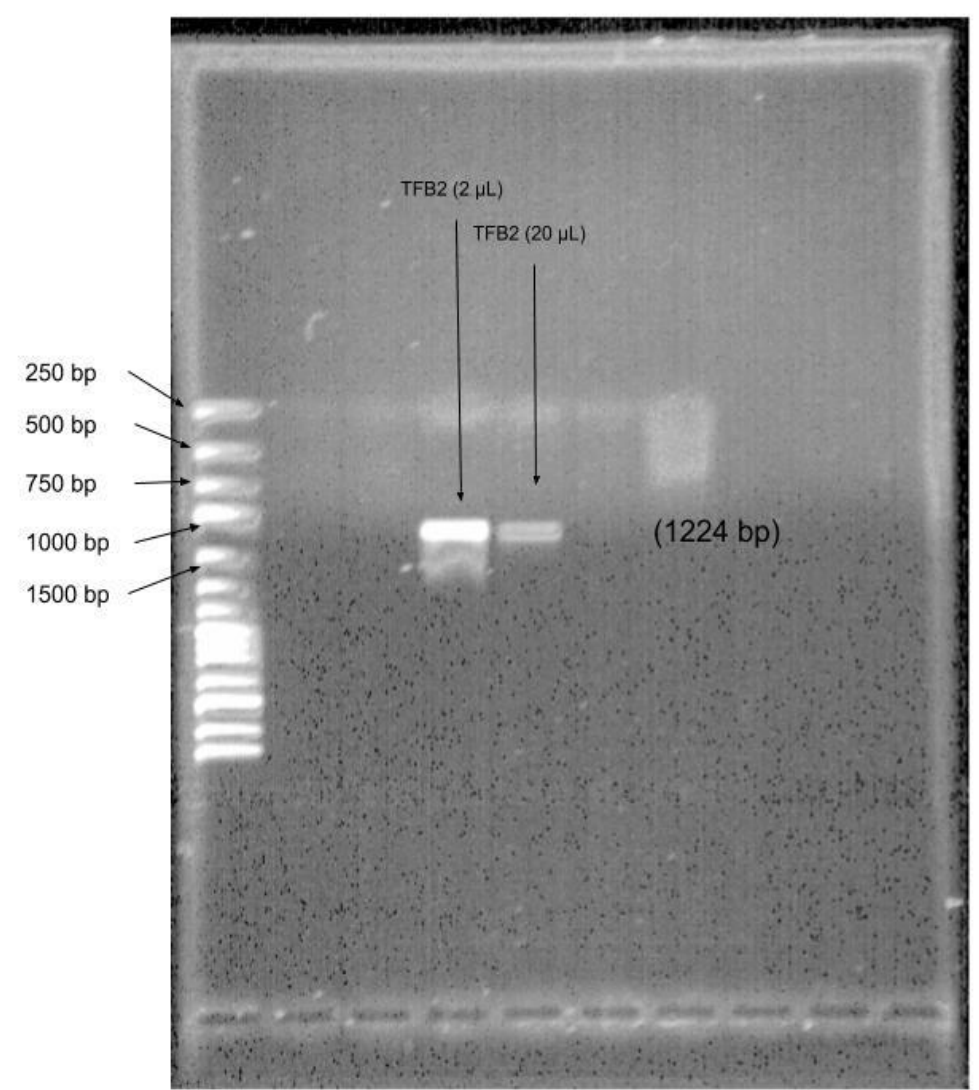

Figure 3: PCR Results for TFB2 Transformation. COM1 Pyrococcus furiosus transformed with TFB2 at both 2 $\mu \mathrm{L}$ and $20 \mu \mathrm{L}$. The O'GeneRuler $1 \mathrm{~kb}$ ladder can be observed on the leftmost side, with indication of transformation shown right around $1224 \mathrm{bp}$. The band indicating $2 \mu \mathrm{L}$ seems to be indicated stronger than the $20 \mu \mathrm{L}$ band. Other lanes for this test were occupied with potential transformants with no indication of successful genetic isolation or PCR viability. 


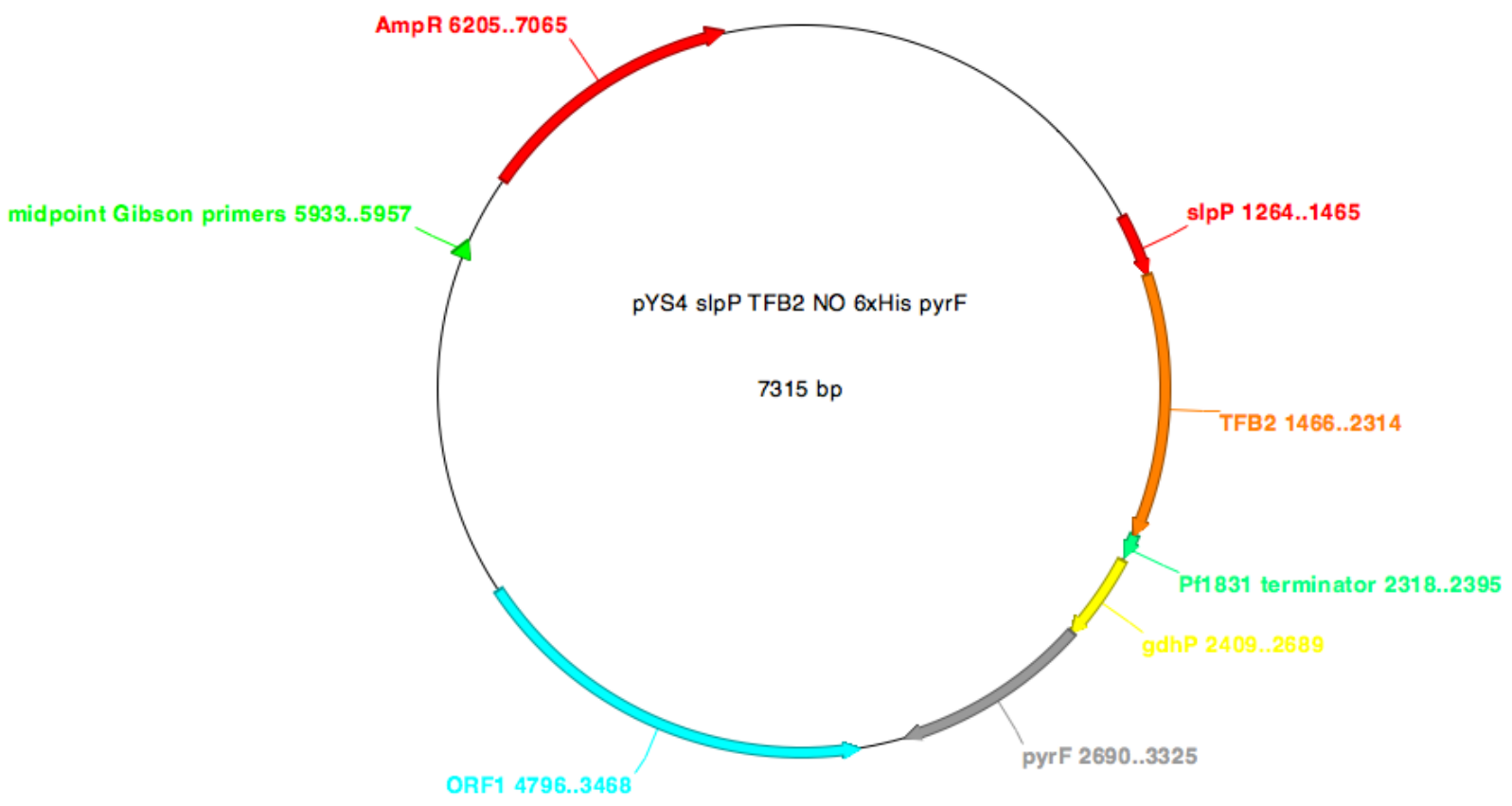

Figure 4: pYS4 TFB2 Overexpression Plasmid. 7315 bp plasmid used in experimentation, developed by Charles Wilson and Michael Bartlett. The pyrF selectable marker can be observed starting at bp 2690, with TFB2 expression occurring at $1466 \mathrm{bp}$.

\section{Discussion:}

The transformation of TFB2 id 228 plasmid into $P$. furiosus COM1, using the pyrF gene for selection, was generally straightforward. TFB2 had better growth, identifiable by greater colony formation in roll tubes and increased opacity in liquid media, over other plasmid transformants being tested around the same time. TGPA and eCGPB plasmids were tested while this experiment was conducted, occupying lanes 1-2 and 4-5 respectively in the PCR check gel. These two plasmids had generally negative results, and have therefore been exempted from any final conclusions.

One exception to the straighforward TFB2 transformation process arose when lack of success in transformation arose around the last month and a half of experimentation in uracil 
negative roll tubes. This apparent lack of transformational competence caused quite a bit of confusion, since transformation occurred so readily in the earlier stages of experimentation,. The situation was likely a result of a faulty COM1 P. furiosus sample that had been stored on the laboratory benchtop for too long. It seems that COM1 has a short period after incubation where the cells most readily uptake DNA in solution. Since the COM1 that proved incompetent for transformation was only around a week and a half old, the window for transformation seems to be most ideal 1-2 days after incubation. Although the evidence is only anecdotal at this point, future experiments attempting to transform plasmids into COM1 should be done as soon as the cells have been removed from the incubator in order to avoid difficulties.

In addition to the difficulty in culturing $P$. furiosus during experimentation, concerns arose during the experimental process regarding the potential accumulation of random mutations in the TFB2 transformants over time. Since the experimental process took a little over 6 months in total, the TFB2 transformants that had been replicated and incubated repeatedly had spent the majority of their lifetime sitting on the benchtop. Mutational accumulation happens to all organisms over enough time, and the cells sitting on the benchtop did not likely come into contact with any materials that would increase their mutational rate. However, in experiments that test the effectiveness of extremely specific mechanisms of transcription, it is paramount to have the cell working as close to 'wild-type', in this case - "stock COM1", as possible in order to acquire accurate results. With these concerns in mind, our results and conclusions shown in this paper should not be negatively altered by random mutational accumulation. However, experiments in the future that aim to determine the functional significance of transcription 
factors like TFB2 should take mutational accumulation into account, creating fresh, stock grown cultures before testing biochemical functions.

During PCR, observable in Fig. 3, it seems as though the electrophoresis gel lane with a lower concentration of transformant genomic DNA used was glowing more prominently, indicating generally stronger expression. This is an interesting observation, since it may be assumed that more initial genetic information would create a more concentrated PCR product. However, it seems that the case presented here is the opposite. One possibility is that there may have been something in the genomic DNA preparation that was inhibitory to the PCR reaction.

Regarding future experimentation to understand the functions of TFB2, the next logical step would be to test the transformed TFB2 overexpression mutants in stressful environments. Farshid Taghizadeh, a previous student that conducted research in the Bartlett Lab, was able to test the viability of TFB2 knock-out COM1 strains in oxidatively stressful conditions. This experiment involved varied levels of hydrogen peroxide solution administered to the normally anoxic growth tubes with the TFB2 knockout present. Using a spectrophotometer, absorbance values were then able to be determined over a period of a few days, accurately documenting the growth ability of COM1 in the absence of TFB2 under oxidatively stressful conditions. An experiment similar to this one may be conducted using TFB2 overexpression mutants in order to gather a more defined picture regarding the role of TFB2 in mediating oxidative stress. 


\section{Acknowledgements:}

Firstly, I would like to thank my family and close friends for supporting me through my experimental lab work for the past half year. While my stress levels in completing my project were often high, I appreciate all the caring people in my life. I would also like to give a huge 'thank you' to the Bartlett Lab for teaching me much more than I could have hoped for. I feel as though my interest in microbial systems has been extended much farther than I previously had thought possible, and the possibility of pursuing a career in research seems infinitely more feasible. 


\section{$\underline{\text { References: }}$}

1. Fiala, G., \& Stetter, K. O. (1986). Pyrococcus furiosus sp. nov. represents a novel genus of marine heterotrophic archaebacteria growing optimally at $100 \mathrm{C}$. Archives of Microbiology, 145(1), 56-61.

2. Kengen, S. W. (2017). Pyrococcus furiosus, 30 years on. Microbial biotechnology, 10(6), 1441-1444.

3. Lipscomb, G. L., Stirrett, K., Schut, G. J., Yang, F., Jenney, F. E., Scott, R. A., ... \& Westpheling, J. (2011). Natural competence in the hyperthermophilic archaeon Pyrococcus furiosus facilitates genetic manipulation: construction of markerless deletions of genes encoding the two cytoplasmic hydrogenases. Appl. Environ. Microbiol., 77(7), 2232-2238.

4. Lundberg, K. S., Shoemaker, D. D., Adams, M. W., Short, J. M., Sorge, J. A., \& Mathur, E. J. (1991). High-fidelity amplification using a thermostable DNA polymerase isolated from Pyrococcus furiosus. Gene, 108(1), 1-6.

5. Micorescu, M., Grünberg, S., Franke, A., Cramer, P., Thomm, M., \& Bartlett, M. (2008). Archaeal transcription: function of an alternative transcription factor B from Pyrococcus furiosus. Journal of bacteriology, 190(1), 157-167.

6. Thorgersen, M. P., Stirrett, K., Scott, R. A., \& Adams, M. W. (2012). Mechanism of oxygen detoxification by the surprisingly oxygen-tolerant hyperthermophilic archaeon, Pyrococcus furiosus. Proceedings of the National Academy of Sciences, 109(45), 1854718552.

7. Williams, E., Lowe, T. M., Savas, J., \& DiRuggiero, J. (2007). Microarray analysis of the hyperthermophilic archaeon Pyrococcus furiosus exposed to gamma irradiation.

Extremophiles, 11(1), 19-29.

8. Woese, C. R., \& Fox, G. E. (1977). Phylogenetic structure of the prokaryotic domain: the primary kingdoms. Proceedings of the National Academy of Sciences, 74(11), 5088-5090. 